\title{
Biomechanical and morphological changes of rabbit corneas under collagenase type II and negative pressure: three months follow-up observation
}

\section{Xinyan Chen}

Capital Medical University

\section{Xiao Qin}

Capital Medical University

Mengyao Yu

Capital Medical University

Haixia Zhang

Capital Medical University

Lin Li ( $\nabla$ lil@ccmu.edu.cn )

Capital Medical University https://orcid.org/0000-0002-9190-5475

Research article

Keywords: biomechanical property, negative pressure, collagenase, ectatic corneas

Posted Date: January 31st, 2020

DOI: https://doi.org/10.21203/rs.2.22389/v1

License: (c) (1) This work is licensed under a Creative Commons Attribution 4.0 International License.

Read Full License 


\section{Biomechanical and morphological changes of rabbit corneas under collagenase type II and negative pressure: three months follow-up observation}

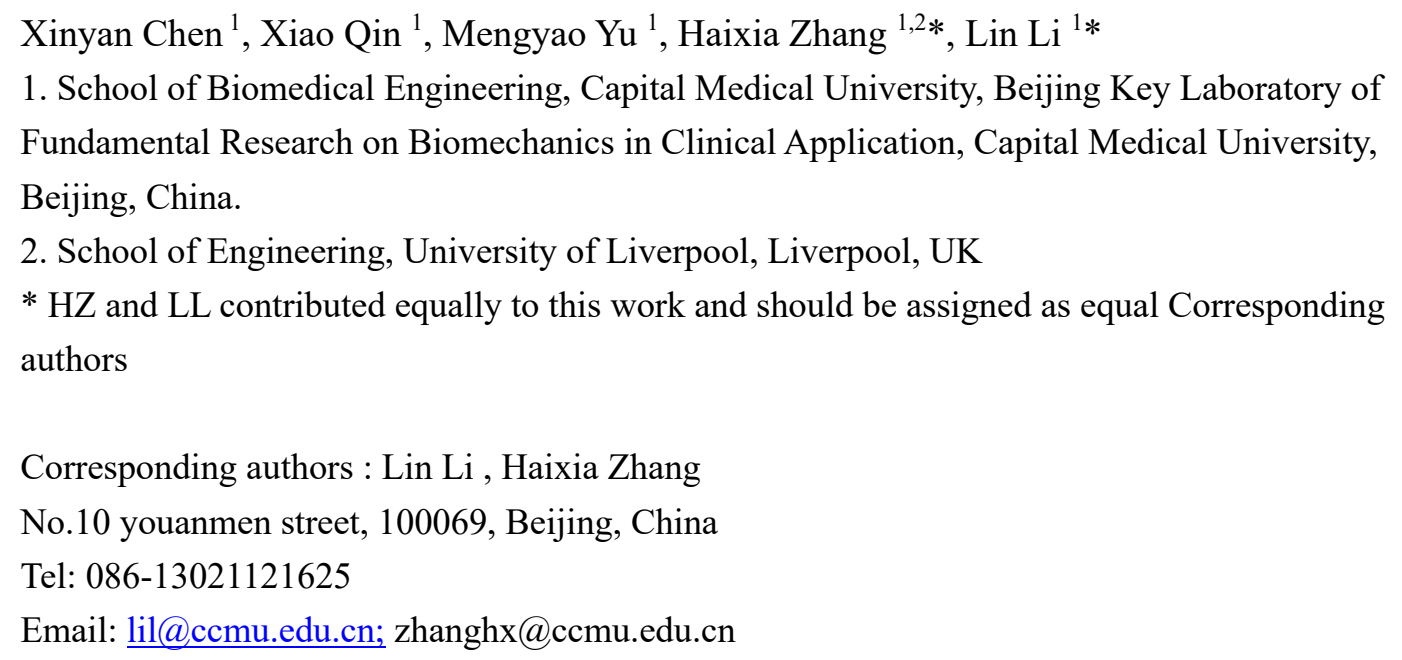

\section{Abstract}

Background: To investigate biomechanical and morphological changes of rabbit cornea ectasia induced by collagenase type II and negative pressure during 3 months after treatment.

Method: Eighteen New Zealand white rabbits were randomly and evenly arranged into three groups. In group NP, the corneas were continuously attracted by negative pressure with $500 \mathrm{mmHg}$ for $30 \mathrm{~min}$, treated by every other day, three times in total. In group CII, the corneal central zone was soaked in the collagenase type II solution (200 $\mu \mathrm{L}$ of $3 \mathrm{mg} / \mathrm{ml}$ ) for $30 \mathrm{~min}$. In group CP, the corneas were disposed as group CII firstly, then applied neg ative pressure as group NP for once after 5 days. All right eyes were treated as control eyes. Corneal morphology and biomechanical related parameters were observed in 
1 vivo once a week for three weeks after treatment and before execution. Histology and

2 biomechanics were tested in vitro at the third month after treatment.

3 Results: Corneal diopter and corneal central thickness (CCT) changed to some extent

4 after treatment immediately as a result of negative pressure in group NP. Three

5 months after treatment, their elastic modulus increased and the relaxation degree

6 decreased compared with the control one. In Group CII, corneal diopter increased,

$7 \mathrm{CCT}$ and $\mathrm{CH}$ decreased at the second week after treatment, which showed the

8 characters of ectatic corneas. At the third month after treatment, elastic modulus $\left(\mathrm{E}_{\mathrm{L}}\right)$

9 of the experimental eyes decreased slightly compared with the control eyes. Due to 10 the large individual differences, there were no regular changes on experimental 11 corneas in Group CP.

12 Conclusions: Cornea socked with collagenase type II showed the obvious 13 characteristics of ectatic cornea at the second week after treatment, but the characteristics disappeared gradually. Negative pressure can result in the change of corneal thickness and diopter in a short period of time, and the degree of swelling and duration are far less than Group CII, but the changes of biomechanical parameters are 17 more obviously than Group CII.

Keywords: biomechanical property; negative pressure; collagenase; ectatic corneas

\section{Background}


1 the ophthalmic refractive system. Corneal refractive index is closely related to the

2 corneal morphology, which is determined by intraocular pressure, corneal

3 biomechanical properties and so on ${ }^{[1]}$. Corneal ectasia is a disease of refractive

4 instability with progressive destruction of corneal structure ${ }^{[2]}$, characterized by

5 central and paracentral corneal stroma thinning and dilation, corneal protrusion,

6 irregular astigmatism and myopia ${ }^{[3,4]}$. Keratoconus (KC) and ectasia after laser

7 corneal refractive surgery is two common diseases of corneal ectasia. $\mathrm{KC}$ is reported

8 approximately $1 / 2000$ in the world, with no sex or race predilection ${ }^{[5]}$. Since the

9 corneal refractive surgery was carried out, ectasia has gained more attention ${ }^{[6]}$.

10 Patients with severe corneal dilatation need corneal transplantation or even blindness,

11 and the pathogenesis of corneal dilatation is still unclear.

An animal model with corneal anatomy and physiology similar to human being is a valuable and indispensable tool in basic research. It provides a possibility for better studying the pathophysiology, observe the pathogenesis and explore treatment methods for corneal ectasia. However, due to the complex etiology, there is no reliable and stable corneal ectasia animal model, and no evaluation criteria for the successful

17 animal model ${ }^{[7]}$. Tachibana et al. ${ }^{[8]}$ constructed spontaneous mutated mice with keratoconus appearance (conical cornea exhibiting apoptosis and elevated expression of c-fos protein in keratocytes) by the genetic tendency of keratoconus. While that kind of corneal ectasia is secondary caused by keratitis, which is caused by genetic mutation. This model demonstrates androgen dependency and its pathogenesis and corneal mechanical properties are different from keratoconus. The post-LASIK (Laser 
1 Assisted In-situ Keratomi) corneal ectasia model is constructed by cutting the corneal

2 matrix ${ }^{[9,10]}$, but not commonly used due to the high modeling cost. Jing $\mathrm{Q}^{[11]}$ made

3 the corneal ectasia rabbit model by degrading corneal collagen, and got the same

4 change of mechanical properties and morphology as keratoconus. But only 14 days in

5 vivo observation was reported. The longer period validity of animal models remain to

6 be further explored.

7 Corneal thickness thinning and local mechanical property weakening are two

8 important factors leading to the cornea ectasia, which have been studied previously ${ }^{[12,}$

9 13]. Additionally, cornea is also usually subjected to external forces, such as eyes rubbing ${ }^{[14]}$, abnormal changes of intraocular pressure. Negative pressure suction is

11 widely used in phacoemulsification, Laser in situ Keratomileusis (LASIK), would drainage and so on. As an external factor, negative pressure attraction may directly cause changes in tissue growth state. Therefore, in this study, we considered two kinds of treatments on cornea, namely negative pressure and collagenase type II solution treatments, to observe the corneal morphological and biomechanical related parameters for three months after treatment.

\section{Methods}

\section{Animals}

Eighteen healthy New Zealand rabbits aged 7 months were selected from the animal department of Capital Medical University. During the observation of the testing machine, all animals were kept in the SPF class animal room of capital 
1

medical university. The protocol for experimental animal was approved according to relevant laws and institutional regulations. All rabbit eyes were examined by slit lamp to exclude anterior segment lesions. The healthy rabbits were randomly divided into three groups, six rabbits for each group. Group NP and CII were treated with negative pressure, and collagenase type II solution, respectively. Group CP were treated with collagenase type II solution and negative pressure in turn. In view of no determined evaluation criteria for the success of corneal ectasia animal model ${ }^{[7]}$, we focus on diopter changes, corneal thickness and biomechanical related parameters reduction, which are regard as the sign of cornea ectasia. After in-vivo observation, the animals were euthanized by overanesthetic injection of $30 \mathrm{~mL} 25 \%$ uratan solution with normal saline through the ear vein for follow-up experiments.

\section{Morphology and biomechanical related parameters}

Keratometer (TOPCON, Japan) and Optical Coherence Tomography (OCT, TOPCON, Japan) were used to get corneal surface curvature along horizontal and vertical meridians. Handheld opthalmotonometer (iCare, Finland) was used to measure intraocular pressure (IOP). Ultrasound Pachymetry (TOMEY, Japan) was used to get central cornea thickness (CCT). Ocular Response Analyzer (ORA, Reichert Inc., Depew, NY) was used to measure biomechanical related parameters: Corneal Resistant Factor (CRF) or Corneal Hysteresis (CH). All left and right corneas of rabbit of three groups were measured on the first, second, third week and third month after treatment.

\section{Negative pressure adsorption process}


2 with the suction pump, $50 \mathrm{~mL}$ needle tubing, pressure sensor, hard connection

3 pipeline and suction catheter. The air in the suction catheter was pumped out by the

4 suction pump, and its value of pressure was measured by the pressure sensor.

5 According to the ideal gas state equation, $P V=\mathrm{nR} T$, the theoretical value of pressure

6 in the suction catheter was calculated, in which the temperature $T$ was the room

7 temperature. By comparing the theoretical and experimental values, the experimental

8 value of pressure in the negative pressure device was calibrated.

9 The anesthetized rabbits were put on the experiment table. $500 \mathrm{mmHg}$ pressures (260 $\mathrm{mmHg}$ less than one atmosphere pressure) were applied to the left cornea with the negative pressure device for 30 minutes. Repeat the same experiment three days in a row. After the experiment, the anti-infection treatment was applied on the eye of rabbit.

\section{Collagenase solution treatment process}

After anesthesia and epithelial debridement, the corneal central zone of left eyes were soaked in the collagenase type II solution $(3 \mathrm{mg} / \mathrm{ml}, 200 \mu \mathrm{L})$. PBS liquid was used afterwards to treat the anti-infection treatment during postoperative 1 week.

\section{Histology}

After 3 months of treatment, 3 rabbits in each group were taken out and their corneas were fixed with $4 \%$ paraformaldehyde for 24 hours, and then embedded with paraffin and stained with hematoxylin-eosin.

\section{Biomechanical measurements}



corneas were cut into a $3 \mathrm{~mm}$-wide strip by a double-edged knife along the

3 nasal-temporal direction. The uniaxial tensile test was performed on the

4 Care-IBTC-50 Testing System (CARE Measurement \& Control Corp, Tianjin, China)

5 in normal saline bath apparatus at room temperature with $25^{\circ} \mathrm{C}$. After preconditioned,

6 the stress-strain test with the tensile rate of $0.02 \mathrm{~mm} / \mathrm{s}$ was carried out, and the

7 stretching amplitude is $115 \%$ of original length. After a five-minute recovery, a

$8 \quad 10$-minute stress-relaxation test was performed afterwards.

9 The stress and strain data of corneal strips were calculated by Eqs. (1-2) from the loading and displacement data obtained in the uniaxial tensile test.

$$
\begin{gathered}
\sigma=F / A_{0} \\
\varepsilon=\Delta l / l_{0}
\end{gathered}
$$

$\sigma$ is the stress, $F$ is the load in uniaxial stretching, and $A_{0}$ is the initial cross-sectional area of the cornea strip. $\varepsilon$ is strain, $\Delta l$ is the displacement of the corneal strips, $l_{0}$ is the initial length of the corneal strips.

According to the literature ${ }^{[15]}$, the stress-strain curves obtained from the uniaxial tensile test in vitro were divided into three regions: low strain region, nonlinear region and high strain region. The nonlinear regions of strain-stress curves were fitted exponentially using Eq. (3), and the low and high strain regions were fitted linearly, respectively.

$$
\sigma=A\left(e^{B \varepsilon}-1\right)
$$

where $A$ and $B$ are model parameters. The tangent modulus is 


$$
\frac{d \sigma}{d \varepsilon}=B \sigma+A B
$$

2 where $B$ is the slope of tangent modulus with stress, we used it to indicate the change

3 of mechanical properties of cornea. The stress relaxation curves were fitted by the

$4 \quad$ second order Prony series model, namely

5

$$
G(t)=1-a_{1}\left(1-e^{-t / \tau_{1}}\right)-a_{2}\left(1-e^{-t / \tau_{2}}\right)
$$

where $G(t)=\sigma(t) / \sigma(0)$ is the normalized stress, relaxation function, $\sigma(t)$ is the stress at time $t, \sigma(0)$ is the initial stress. $a_{1}, a_{2}, \tau_{1}$ and $\tau_{2}$ are stress relaxation parameters.

\section{Result}

\section{In vivo tests}

We got corneal surface curvature along horizontal and vertical directions $\left(\mathrm{CC}_{-} \mathrm{H}\right.$ and $\left.\mathrm{CC}_{-} \mathrm{V}\right), \mathrm{CCT}$, biomechanical related parameters ( $\mathrm{CH}$ and $\left.\mathrm{CRF}\right)$ of three groups at the first, second, third week and third month after treatment. To better describe the changes of the measurement quantities, we defined the change of them. For example, the change of CCT $(\triangle \mathrm{CCT})$ is as follows:

$$
\Delta \mathrm{CCT}=\mathrm{CCT}_{\text {post-treatment }}-\mathrm{CCT}_{\text {pre-treatment }}
$$

$\Delta \mathrm{CCT}$ of these three groups were showed in Fig. 2. $\Delta \mathrm{CCT}$ of experimental eyes in Group NP (Negative pressure group) increased at the first week after treatment, and then decreased slightly (Fig. 2a). The results of the paired T test showed no significant difference between experimental eyes before and after treatment $(p=0.063>0.05)$, and between experimental eyes and control eyes $(p=0.198>0.05)$ after three months. 
1 The peripheral corneal thickness (PCT) of experimental eyes had the same trend with

2 CCT.

In Group CII, from Fig. 2b, $\Delta$ CCT of experimental eyes obviously decreased at the second week after treatment, which was statistically significant compared with pre-treatment and the control group $(p=0.003<0.05 ; p=0.01<0.05)$. After 3 weeks, $\Delta$ CCT was recovered gradually, and there was no significant difference between the experimental and control one $(\mathrm{p}=0.468>0.05)$ at the third month after treatment.

In Group CP, form Fig. 2c, the mean values of CCT for experimental eyes were slightly thicker during 1 to 3 weeks and then recovered. But the changes were not statistically significant compared with pre-treatment and control group $(\mathrm{p}=0.507>$ $0.05 ; \mathrm{p}=0.748>0.05)$. The large standard deviation for each experimental time point showed that the individual difference was significant.

The variations of corneal surface curvature along horizontal and vertical directions $\left(\Delta \mathrm{CC}_{-} \mathrm{H}\right.$ and $\left.\Delta \mathrm{CC}_{-} \mathrm{V}\right)$ were shown in Fig. 3 . We used $\mathrm{D}_{\mathrm{H}}$ and $\mathrm{D}_{\mathrm{V}}$ to represent the diopter of cornea along horizontal and vertical direction respectively.

In the Group NP, from Fig. $3 \mathrm{a}$ and $3 \mathrm{~d}, \Delta \mathrm{CC}_{-} \mathrm{H}$ and $\Delta \mathrm{CC}_{-} \mathrm{V}$ fluctuated slightly after the treatment. CC_H was significantly different between pre- and post-treatment at the third week $(p=0.039<0.05)$, while $C_{-} V$ was not $(p=0.078>0.05)$. We noted that the diopter of cornea along horizontal and vertical directions $\left(\mathrm{D}_{\mathrm{H}}\right.$ and $\left.\mathrm{D}_{\mathrm{V}}\right)$ decreased by $1.42 \mathrm{D}$ and $1.91 \mathrm{D}$ respectively at the third month after treatment.

In Group CII, from Fig. $3 \mathrm{~b}$ and $3 \mathrm{e}$, the mean values of $\Delta \mathrm{CC}_{-} \mathrm{H}$ showed an obviously decrease at the first week, and then recovered at the third week after 
1 treatment. $\mathrm{D}_{\mathrm{H}}$ at the first week increased by $4.38 \mathrm{D}$ compared with pre-treatment, and

2 4.10D compared with control group. As to CC_V, there was no obvious change after treatment.

In the Group CP, from Fig. $3 \mathrm{c}$ and $3 \mathrm{f}, \Delta \mathrm{CC}_{-} \mathrm{H}$ and $\Delta \mathrm{CC}_{-} \mathrm{V}$ increased at the first week and then showed a trend of gradual growth. After three months, the diopter of the experimental eye $\left(\mathrm{D}_{\mathrm{H}}\right.$ and $\left.\mathrm{D}_{\mathrm{V}}\right)$ was larger than those of the control eye by $1.83 \mathrm{D}$ and 2.21D. Paired T test shows that there was no significant difference between preand post-treatment of experimental eyes $\left(p=0.533>0.05\right.$ of $\mathrm{CC}_{-} \mathrm{H}, \mathrm{p}=0.739>0.05$ of $\left.\mathrm{CC}_{-} \mathrm{V}\right)$, as well as experimental eyes and control eyes $\left(\mathrm{p}=0.511>0.05\right.$ of $\mathrm{CC}_{-} \mathrm{H}, \mathrm{p}$ $=0.811>0.05$ of $\mathrm{CC}_{-} \mathrm{V}$ ) at the third month after treatment.

Corneal Hysteresis $(\mathrm{CH})$ and Corneal Resistance Factor (CRF), the output parameter of ORA, were used to observe the changes of corneal mechanical properties in vivo after treatment. The changes of $\mathrm{CH}$ and $\mathrm{CRF}$ for three experimental groups were shown in Fig. 4.

For these three groups, $\Delta \mathrm{CH}$ and $\triangle \mathrm{CRF}$ fluctuated around zero, the mean values of variation were lower than $1 \mathrm{mmHg}$. In Group NP, $\Delta \mathrm{CH}$ increased slightly after treatment, and then recovered, while $\triangle \mathrm{CRF}$ decreased slightly and then recovered. In Group CII, as shown in Fig. 4b, the mean values of $\Delta \mathrm{CH}$ for experimental cornea were essentially unchanged, except at the second week after treatment, which significantly decreased compared with that of pre-treatment $(\mathrm{p}=$ $0.021<0.05)$, but there are no significant difference with control eye $(p=0.345>$ $0.05)$. 


\section{Uniaxial tensile tests}

The uniaxial stretching experiment was carried out at the third month after treatment. The stress-strain curves and stress relaxation curves obtained from uniaxial stretch test of corneal strips were shown in Fig 5. The mechanical parameters gained by curves fitting were shown in Fig. $6 . \mathrm{E}_{\mathrm{L}}$ and $\mathrm{E}_{\mathrm{H}}$ were the elastic modulus of cornea at the low- and high-strain region, parameter $B$ were obtained by exponential fitting of stress-strain curves in its nonlinear region. Stress relaxation time $(\tau)$ was defined as the time over which the stress was relaxed halfway between its initial and equilibrium value $^{[165]}$, and relaxation limit $G(\infty)$ was the normalized stress as time was infinity. The results showed that the partition fitting method can better describe the stress-strain curve $\left(\mathrm{R}^{2}>0.98\right)$, and the second order Prony model gave a good fit to the stress relaxation data $\left(\mathrm{R}^{2}>0.99\right)$. Moreover, biomechanical parameters of control corneas were basically consistent with the previous literature on healthy rabbit corneas ${ }^{[17]}$.

From Fig. 6, as to the cornea treated with negative pressure (group NP), we noted that their elastic modulus at low and high stress region $\left(E_{L}\right.$ and $\left.E_{H}\right)$ increased obviously, while conversely in parameter $B$, the slope of tangent modulus with stress. The stress relaxation time increased compared with its controls, it means that the relaxation stress of cornea treated with negative pressure became slow down.

In both Group CII and CP, all the mechanical parameters only showed slightly differences between experimental and control corneas. Further Fig. 6 shows that the differences of mechanical parameters between experimental and control corneas were 
larger in Group CP than those in Group CII.

\section{Histology}

In addition, HE staining was performed to observe the changes in tissue state, and to further confirm the biomechanical change of tissue. As shown in Fig. 7, the structure of each layer was intact both in experimental and control corneas, no obvious abnormality in the cell morphology and no inflammatory cell infiltration was observed, and some epithelial cells were lost due to sectioning. In Group NP, there was no significant difference between the experimental cornea and its control. In Group CII and Group CP, compared with control corneas, the experimental corneas tissue sections showed loose and disordered collagenous fibers, widened interlamellar clefts, curled fibers and some fibers were broken.

\section{Discussion}

Corneal ectasia leading to a decline in quality of life ${ }^{[18]}$, and it is a leading indication for corneal transplantation ${ }^{[17]}$. Although many efforts has been paid to find suitable in vivo corneal ectasia animal model which can deepen the understanding of this disease recent years, there still no uniform direction and method due to its complex pathogenesis. Clinical manifestation of corneal ectasia include thinning, lordosis and ectasia of the central or paracentral corneal stroma, irregular astigmatism, myopia and so on. Corneal thickness, weaken of corneal mechanical properties and external factors are believed to play an important role in the development of ectatic diseases like keratoconus and post-refractive surgery ectasia. In this study, three 
1

treatments (negative pressure adsorption, collagenase type II solution, and used both) were performed in rabbits of three groups respectively. Then experimental rabbits were observed for three months, in order to explore whether treatments could result in and maintain corneal ectasia.

Negative pressure will cause the increase of IOP, and its fluctuation value can be up to $80 \sim 230 \mathrm{mmHg}{ }^{[19,20]}$. High IOP can cause swelling of corneal epithelial edema and matrix, and lead to increased corneal thickness ${ }^{[21]}$. This may be the reason for the immediately significant increase of CCT in the experimental eyes after treatment at the first week. The subsequent trend of decrease may be due to the gradual recovery of stromal edema.

$\mathrm{D}_{\mathrm{H}}$ and $\mathrm{D}_{\mathrm{V}}$ of experimental corneas in Group NP decreased 1.42D and 1.91D respectively at the third month after treatment, and the control corneas decreased 1.30D in both two directions. Studies have shown that the radius of curvature of rabbits increases with the growth and development ${ }^{[22]}$, nearly $0.015 \mathrm{~mm}$ per week ${ }^{[23]}$, namely the corneal diopter of normal rabbits will decrease about 1.00D after three months, which is basically consistent with the changes in control corneas. It indicated that the negative pressure has a little effect on corneal geometry after a three-month recovery. However, the result of mechanical test of corneal strip in vitro showed that the elastic modulus and relaxation behavior of experimental corneas have a great changes. Therefore, the effect of negative pressure on cornea deserves further attention.

In this study, different degrees of swelling were observed in experimental eyes 
1 after treatment with Collagenase type II. At the second week after treatment, an

2 increase of 4.38D in $\mathrm{D}_{\mathrm{H}}$ and decrease of $\mathrm{CCT}$ and $\mathrm{CH}$ were observed in experimental

3 corneas, which is consistent with the clinical manifestations of corneal ectasia, and

4 these results were also consistent with Yan's result ${ }^{[11]}$. It indicated that Collagenase

5 type II does cause temporary changes in the morphological and mechanical properties

6 of the cornea. However, we noticed that $\mathrm{CCT}, \mathrm{CC}_{-} \mathrm{H}, \mathrm{CC}_{-} \mathrm{V}$ and $\mathrm{CH}$ showed a trend

7 of recovery, and the characteristics of ectatic corneas were not observed from the third

8 week to the third month after treatment. Moreover, mean values of elastic modulus $E_{\mathrm{L}}$

9 and $\mathrm{E}_{\mathrm{H}}$ of experimental cornea were slight smaller than that of the controls. Therefore,

10 we believe that the treatment with Collagenase type II can result in ectatic corneas in 11 a short time, and it cannot last a long term.

Stroma is the main bearing part of corneal mechanics, and the damaged cornea may protrusion under intraocular pressure. Collagenase type II is a nonspecific collagenase, which dissolved a part of corneal stroma and resulted in the thinning of the corneal matrix ${ }^{[24]}$. This may explain why the character of ectatic corneas were observed in the short term after treatment. At third month after treatment, HE staining results of corneal sections showed loose and disordered collagenous fibers, widened interlamellar clefts, curled fibers and some fibers were broken, which were consistent with previous literatures ${ }^{[11]}$. The change in fiber structure was consistent with the slightly decreased elastic modulus $\mathrm{E}_{\mathrm{L}}$ and $\mathrm{E}_{\mathrm{h}}$. Therefore, we can deduce that although treated with collagenase might not be an effective way to establish a stable animal model with corneal ectasia, it can lead to weakening tensile mechanical properties of 
the cornea.

Experimental corneas in Group CP showed increased curvature, decreased CRF at the first week after treatment. $\mathrm{D}_{\mathrm{H}}$ and $\mathrm{D}_{\mathrm{V}}$ also decreased $0.83 \mathrm{D}$ and $1.55 \mathrm{D}$ in their control corneas at the third month after treatment respectively, while curvature of experimental corneas did not increase actually as Group CII did. Due to the large individual differences, there were no regular changes on experimental corneas in Group CP. Contrary to cornea treated by collagenase type II, the elastic moduli of corneas treated by negative pressure (Group NP and CP) increased, and its relaxation was slow down. It indicated that the negative pressure on either the collagenase treated or normal corneas would increase the tensile mechanical properties of the cornea.

One limitation of this study was that the negative pressure adsorption modeling method of Group NP only considers the external factors, did not combine with the clinic. The other was the number of specimens used for uniaxial stretching was relatively small.

\section{Conclusion}

In conclude, Collagenase type II results in ectatic corneas around two weeks after treatment, but the degree of swelling decreased with time, and there was no significant difference after three months. Negative pressure results in corneal thickness and diopter change in short time, while the degree of swelling and duration are far less than collagenase type II. The production of cornea ectasia by protease and/or negative pressure adsorption still needs longer observation and further study. 


\section{Abbreviations}

3 Group NP: Group treated with negative pressure

4 Group CII: Group treated with collagenase type II

5 Group CP: Group treated with collagenase type II and negative pressure

6 CCT: Corneal central thickness

$7 \quad$ KC: Keratoconus

8 LASIK: Laser Assisted In-situ Keratomi

9 CRF: Corneal Resistant Factor

CH: Corneal Hysteresis

CC_H: Curvature along horizontal directions

CC_V: Curvature along vertical directions

PCT: The peripheral corneal thickness

$\mathbf{D}_{\mathbf{H}}$ : Diopter of cornea along horizontal direction

$\mathbf{D}_{\mathbf{v}}$ : Diopter of cornea along vertical direction

$\mathbf{E}_{\mathbf{L}}$ : Elastic modulus of cornea at the low-strain region

$\mathbf{E}_{\mathbf{H}}$ : Elastic modulus of cornea at the high-strain region

\section{Declarations}

\section{Ethics approval and consent to participate}

This study was approved by the Ethics Committee at animal department of capital medical university. All the procedures adhered to the regulations of the science and 
1 technology commission of China, the regulations on the control of experimental

2 animals and the ARVO statement on animal experiments in international ophthalmic

3 and visual science research.

4 Consent for publication

$5 \quad$ Not applicable.

6 Availability of data and materials

7 The datasets used and/or analyzed during the current study are available from the

8 corresponding author on reasonable request.

\section{Competing interests}

10 The authors declare that they have no competing interests.

11 Funding

12 This study was supported by National natural science foundation of China 13 (No.31470914, 31370952). The funding agencies had no role in the research design or 14 conduct.

\section{Authors' contributions}

$\mathrm{XC}$ acquired, analyzed data, was a major contributor in writing the manuscript. $\mathrm{XQ}$ performed the ORA examination. MY performed the histological examination of experimental animals. HZ and LL designed the work, interpreted the data and revised the manuscript. All authors read and approved the final manuscript. Acknowledgements

Not applicable. 


\section{References}

2 [1] Edmund C. Corneal elasticity and ocular rigidity in normal and keratoconic eyes.

$3 \quad$ Acta Ophthalmologica. 2010; 66(2):134-140.

4 [2] Dupps DJ Jr. Biomechanical modeling of corneal ectasia. Journal of Refractive $5 \quad$ Surgery. 2005; 21(2):186-190.

6 [3] Krachmer JH, Feder RS, Belin MW. Keratoconus and related noninflammatory corneal thinning disorders. Surv Ophthalmol. 1984; 28(4):293-322.

8 [4] Binder PS, Lindstrom RL, Stulting RD, Donnenfeld E, Wu H, McDonnell P, Rabinowitz Y. Keratoconus and corneal ectasia after LASIK. Journal of Cataract \& Refractive Surgery.2005; 31(11):2035-2038.

[5] Ambekar R, Toussaint KC, Wagoner JA. The effect of keratoconus on the

[9] Seiler T, Koufala K, Richter G. Iatrogenic keratectasia after laser in situ 

keratomileusis. Journal of Refractive Surgery. 1998; 27(4):312-317.

2 [10] Thomas K. Iatrogenic keratectasia: current knowledge, current measurements. 3 Journal of Cataract \& Refractive Surgery. 2002; 28(12):2065-2066.

4 [11]Qian J, Li H, Tang Y, Song W, Rong B, Yang S, Wu Y, Yan X. A rabbit model of corneal Ectasia generated by treatment with collagenase type II. Bmc Ophthalmology. 2018; 18(1):94-100.

[12]Estrada AV, Díez PS, Alió JL. Keratoconus Grading and Its Therapeutic Implications. 2017; 177-184. 

in keratoconus. Cornea. 2017; 36:131-137.

2 [19]Farash SG, Azar DT, Gurdal C, Johnny W. Laser in situ Keratomileusis: literature 3 review of a developing technique. J cataract surg. 1998; 24:989-1006.

4 [20]Ytteborg J, Dohlman CH. Corneal edema and intraocular pressure. II. $5 \quad$ Clinicalresults. Archives of Ophthalmology. 1965; 74(4):477-484.

6 [21]Mackiewicz ZM, Maatta M, Stenman L, Konttinen T, TervoY, Konttinen. 7 Collagenolytic proteinases in keratoconus. American Journal of Ophthalmology. 2006; 142(6):1096-1097.

[22] Roth N. Relationship between corneal radius of curvature and age in rabbits. British Veterinary Journal. 1969; 125(11):560-563.

[23] Wachtlin J, Sehrunder S, Pham DI, Hoffmann F, Hartmann C. Rise in intraocular 


\section{Figures}

2

3 Fig. 1 Diagram of the negative pressure device. The device is made up of the suction pump, $50 \mathrm{~mL}$ needle tubing, pressure sensor, hard connection pipeline and suction catheter. The air inside the device can be sucked out by suction pump to create a negative pressure environment. (6)

Fig. 2 The change of CCT $(\triangle \mathrm{CCT})$. (a) is negative pressure group (Group NP), $\triangle \mathrm{CCT}$ of experimental eyes increased and then decreased slightly, which was no statistically significant compared with pre-treatment and the control group $(\mathrm{p}=0.063>0.05 ; \mathrm{p}=0.198>0.05)$ after three months. (b) is group treated with collagenase type II (Group CII), $\Delta$ CCT of experimental eyes obviously decreased at the second week after treatment, and significantly different from pre-treatment and the control group $(\mathrm{p}=0.003<0.05 ; \mathrm{p}=0.01<0.05)$. Then $\Delta$ CCT of experimental eyes recovered gradually and no significant differ from the control one $(p=0.468>$ 0.05 ) at the third month after treatment. (c) is group treated with such two disposals, the mean values of CCT for experimental eyes were slightly thicker and then recovered, not statistically significant was observed compared with pre-treatment and control group $(\mathrm{p}=0.507>0.05 ; \mathrm{p}=$ $0.748>0.05$ ). The solid points are experimental data, and the hollowed points are control one.

Fig. 3 The change of corneal surface curvature for three groups. (a-c) and (d-f) are CC along the horizontal and vertical direction $\left(\Delta \mathrm{CC}_{-} \mathrm{H}\right.$ and $\left.\Delta \mathrm{CC}_{-} \mathrm{V}\right)$, respectively. In the Group NP, CC_H (a) was slight fluctuation post-treatment, and significantly differ from the pre-treatment at the third week $(p=0.039<0.05)$, while CC_V $(d)$ was not $(p=0.078>0.05)$. In Group CII, the mean 
values of $\Delta \mathrm{CC}_{-} \mathrm{H}$ (b) obviously decreased at the first week and then recovered, no obvious change on $\mathrm{CC}_{-} \mathrm{V}(\mathrm{e})$. In the Group $\mathrm{CP}, \Delta \mathrm{CC}_{-} \mathrm{H}$ (c) and $\Delta \mathrm{CC}_{-} \mathrm{V}$ (f) increased immediately and then gradual growth, there was no significant difference between pre- and post-treatment of experimental eyes $\left(p=0.533>0.05\right.$ of $\mathrm{CC}_{-} \mathrm{H}, \mathrm{p}=0.739>0.05$ of $\left.\mathrm{CC} \_\mathrm{V}\right)$, as well as experimental eyes and control eyes $\left(p=0.511>0.05\right.$ of $\mathrm{CC}_{-} \mathrm{H}, \mathrm{p}=0.811>0.05$ of $\left.\mathrm{CC}_{-} \mathrm{V}\right)$ at the third month after treatment.

(1)

Fig. 4 The changes of corneal mechanical properties in vivo for three groups. (a-c) and (d-f) are $\Delta \mathrm{CH}$ and $\Delta \mathrm{CRF}$, respectively. In Group NP, $\Delta \mathrm{CH}$ (a) increased slightly and then recovered, while $\triangle \mathrm{CRF}$ (d) first decreased slightly. In Group CII, the mean values of $\Delta \mathrm{CH}$ (b) for experimental cornea were significantly decreased compared with that of pre-treatment $(p=0.021<0.05)$, while no significant difference with control eye $(p=0.345>0.05)$ at the second week after treatment.

Fig. 5 Stress-strain curves (a) and normalized stress relaxation curves (b) of corneal strips. These curves will be fitting to obtain mechanical parameters, $\mathrm{E}_{\mathrm{L}}, \mathrm{E}_{\mathrm{H}}$ and parameter $B$ were obtained by stress-strain curves. Stress relaxation time $(\tau)$ and relaxation limit $G(\infty)$ were obtained by normalized stress relaxation curves.

Fig. 6 The biomechanical parameters of cornea in three groups. (a) and (c) are the results of the elastic modulus in the low and high stress region of strain-stress curves, (b) is the results of parameter $B$ in the nonlinear region of strain-stress curves, (d) and (e) are the results of stress relaxation limit and relaxation time of corneas. In group NP, elastic modulus at low and high stress 
1 region $\left(\mathrm{E}_{\mathrm{L}}\right.$ and $\mathrm{E}_{\mathrm{H}}$, a and $\mathrm{c}$ ) increased obviously, while conversely in parameter $B(\mathrm{~b})$. And the

2 stress relaxation time (e) increased, which indicated that the relaxation stress of experimental

3 corneas became slow down. In both Group CII and CP, no significant difference was observed in

$4 \quad$ all the mechanical parameters between experimental and control corneas, and the differences in

5 Group CP were slightly larger than those in Group CII.

6

7 Fig. 7 Hematoxylin-eosin stained corneal sections. The structure of each layer was intact and no

8 obvious abnormality in the cell morphology was observed in all group. Some epithelial cells were

9 lost due to sectioning. No significant difference between the experimental and control corneas in

Group NP (the first row), while the experimental corneas tissue sections showed loose and disordered collagenous fibers, widened interlamellar clefts compared with control corneas in Group CII and Group CP (the second and third row). 


\section{Figures}

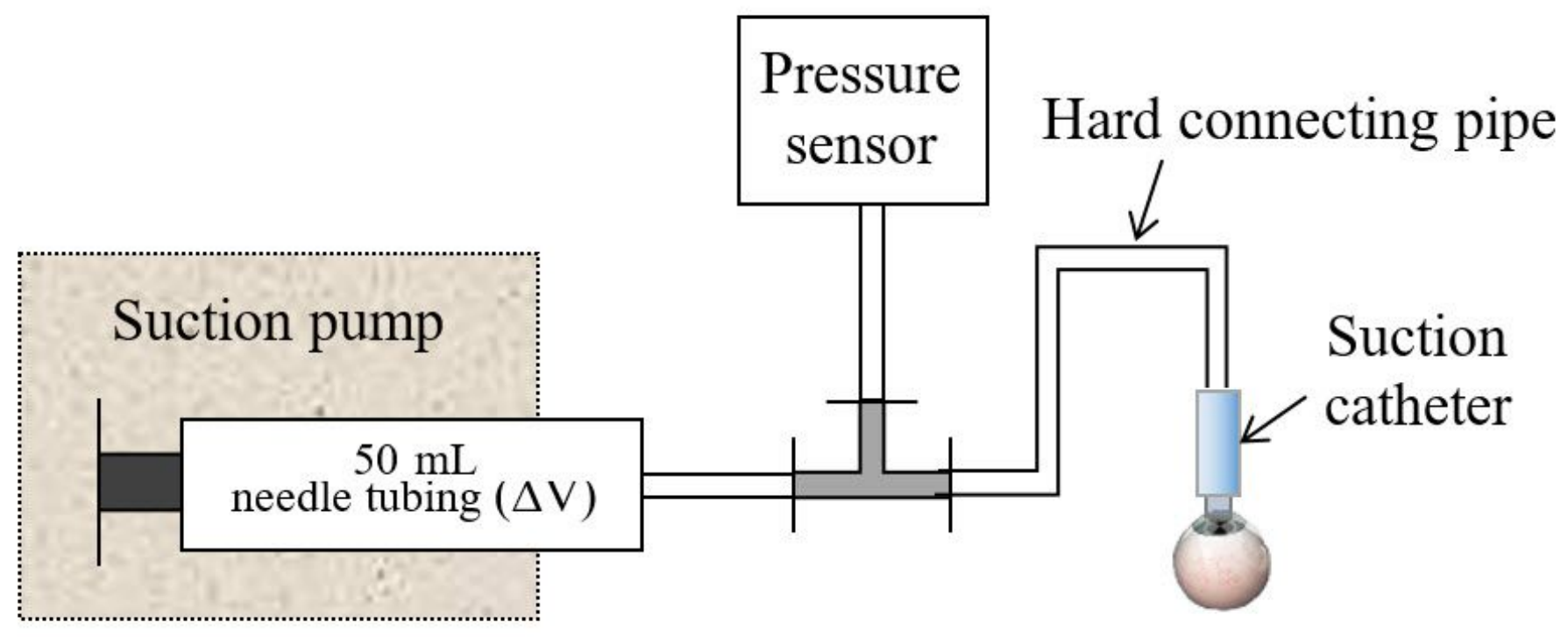

Figure 1

Diagram of the negative pressure device. The device is made up of the suction pump, $50 \mathrm{~mL}$ needle tubing, pressure sensor, hard connection pipeline and suction catheter. The air inside the device can be sucked out by suction pump to create a negative pressure environment.
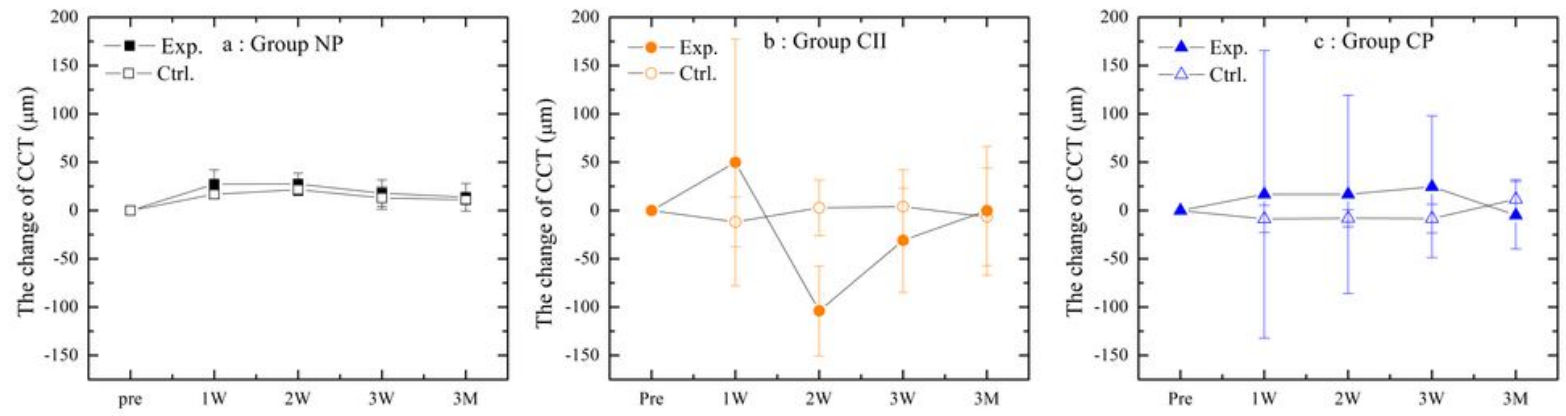

\section{Figure 2}

The change of CCT $(\triangle \mathrm{CCT})$. (a) is negative pressure group (Group NP), $\triangle \mathrm{CCT}$ of experimental eyes increased and then decreased slightly, which was no statistically significant compared with pre-treatment and the control group $(p=0.063>0.05 ; p=0.198>0.05)$ after three months. $(b)$ is group treated with collagenase type II (Group CII), $\triangle \mathrm{CCT}$ of experimental eyes obviously decreased at the second week after treatment, and significantly different from pre-treatment and the control group $(p=0.003<0.05 ; p=0.01$ $<0.05)$. Then $\triangle C C T$ of experimental eyes recovered gradually and no significant differ from the control one $(p=0.468>0.05)$ at the third month after treatment. (c) is group treated with such two disposals, the mean values of CCT for experimental eyes were slightly thicker and then recovered, not statistically 
significant was observed compared with pre-treatment and control group $(p=0.507>0.05 ; p=0.748>$ 0.05). The solid points are experimental data, and the hollowed points are control one.
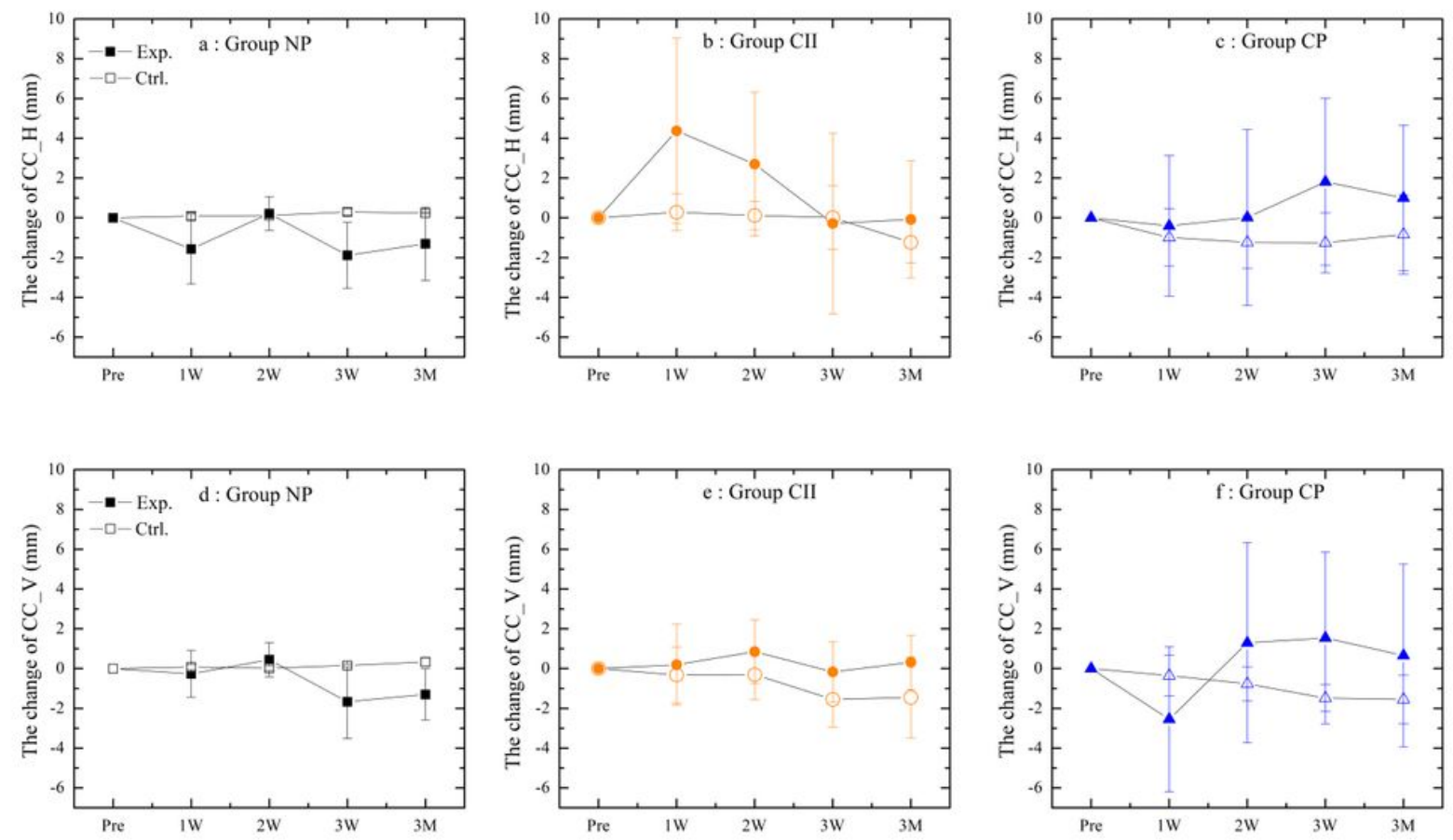

\section{Figure 3}

The change of corneal surface curvature for three groups. (a-c) and (d-f) are CC along the horizontal and vertical direction ( $\triangle \mathrm{CC} \_\mathrm{H}$ and $\triangle \mathrm{CC} \_\mathrm{V}$ ), respectively. In the Group NP , CC_H (a) was slight fluctuation posttreatment, and significantly differ from the pre-treatment at the third week $(p=0.039<0.05)$, while CC_V (d) was not $(p=0.078>0.05)$. In Group Cll, the mean values of $\triangle \mathrm{CC} \_\mathrm{H}$ (b) obviously decreased at the first week and then recovered, no obvious change on CC_V(e). In the Group CP, $\triangle \mathrm{CC} \_H$ (c) and $\triangle \mathrm{CC} \_\mathrm{V}(\mathrm{f})$ increased immediately and then gradual growth, there was no significant difference between pre- and post-treatment of experimental eyes $\left(p=0.533>0.05\right.$ of $\mathrm{CC} \_\mathrm{H}, \mathrm{p}=0.739>0.05$ of $\left.\mathrm{CC} \_\mathrm{V}\right)$, as well as experimental eyes and control eyes $\left(p=0.511>0.05\right.$ of $C_{-} \_H, p=0.811>0.05$ of CC_V $)$ at the third month after treatment. 

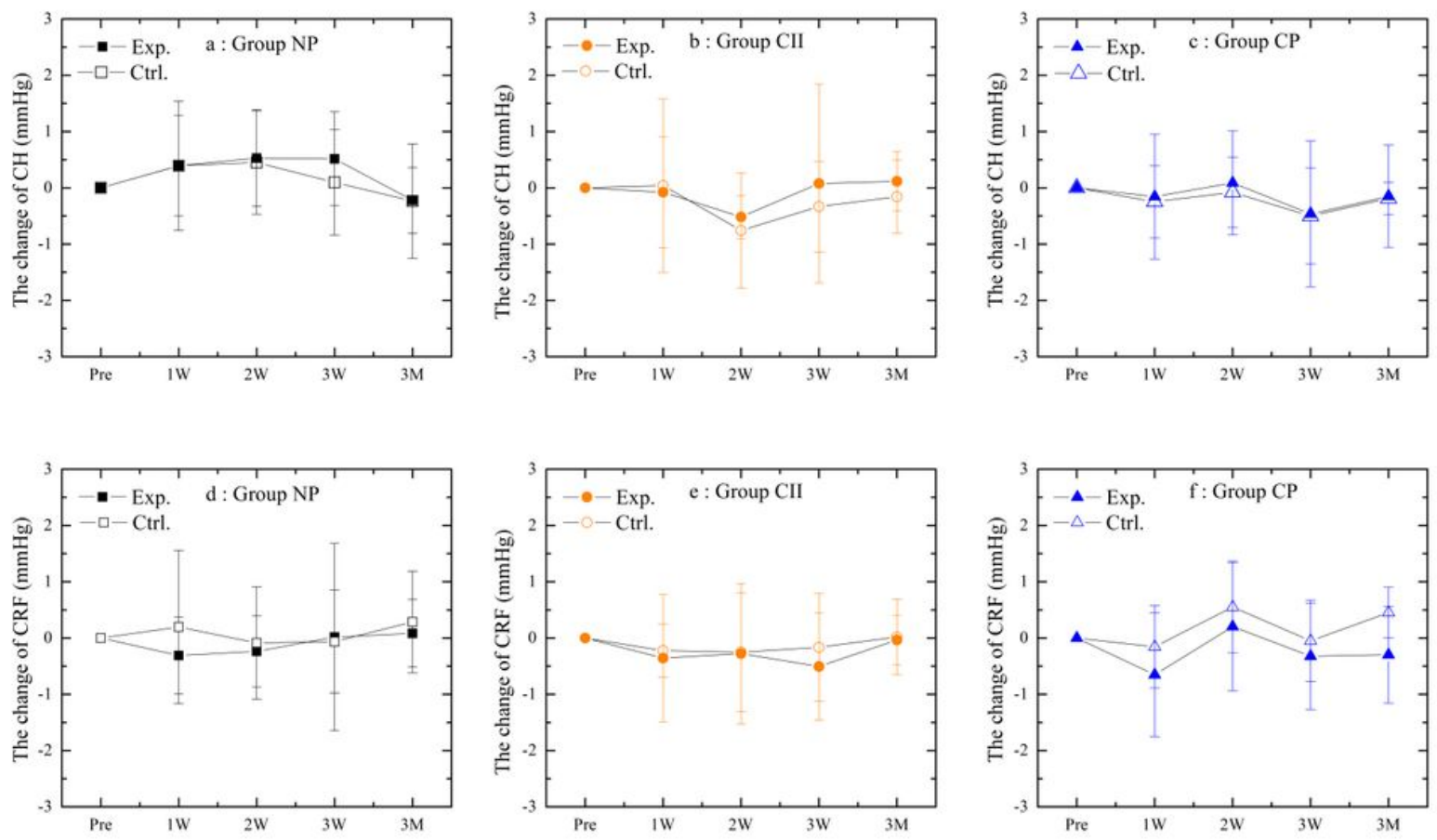

\section{Figure 4}

The changes of corneal mechanical properties in vivo for three groups. (a-c) and (d-f) are $\Delta \mathrm{CH}$ and $\Delta \mathrm{CRF}$, respectively. In Group NP, $\triangle \mathrm{CH}$ (a) increased slightly and then recovered, while $\Delta \mathrm{CRF}$ (d) first decreased slightly. In Group $\mathrm{Cll}$, the mean values of $\Delta \mathrm{CH}(\mathrm{b})$ for experimental cornea were significantly decreased compared with that of pre-treatment $(p=0.021<0.05)$, while no significant difference with control eye $(p$ $=0.345>0.05)$ at the second week after treatment.
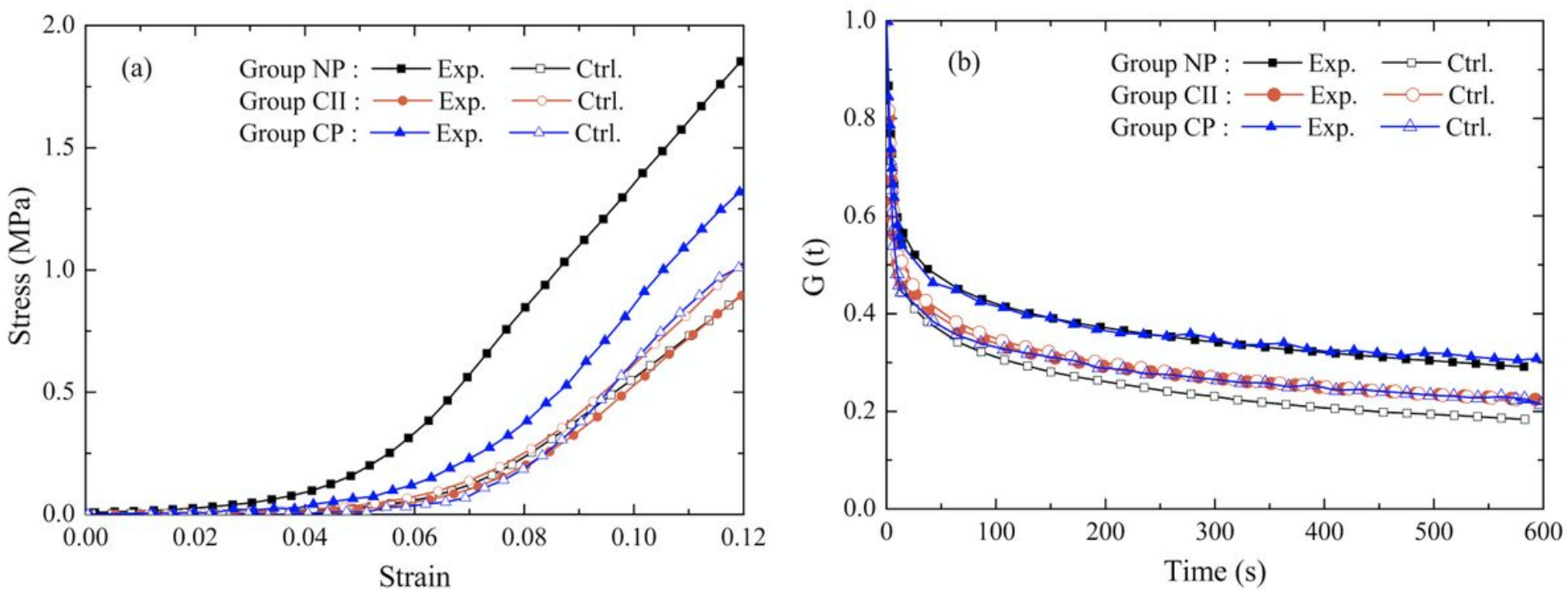

Figure 5

Stress-strain curves (a) and normalized stress relaxation curves (b) of corneal strips. These curves will be fitting to obtain mechanical parameters, EL , EH and parameter B were obtained by stress-strain curves. 
Stress relaxation time $(\tau)$ and relaxation limit $\mathrm{G}(\infty)$ were obtained by normalized stress relaxation curves.
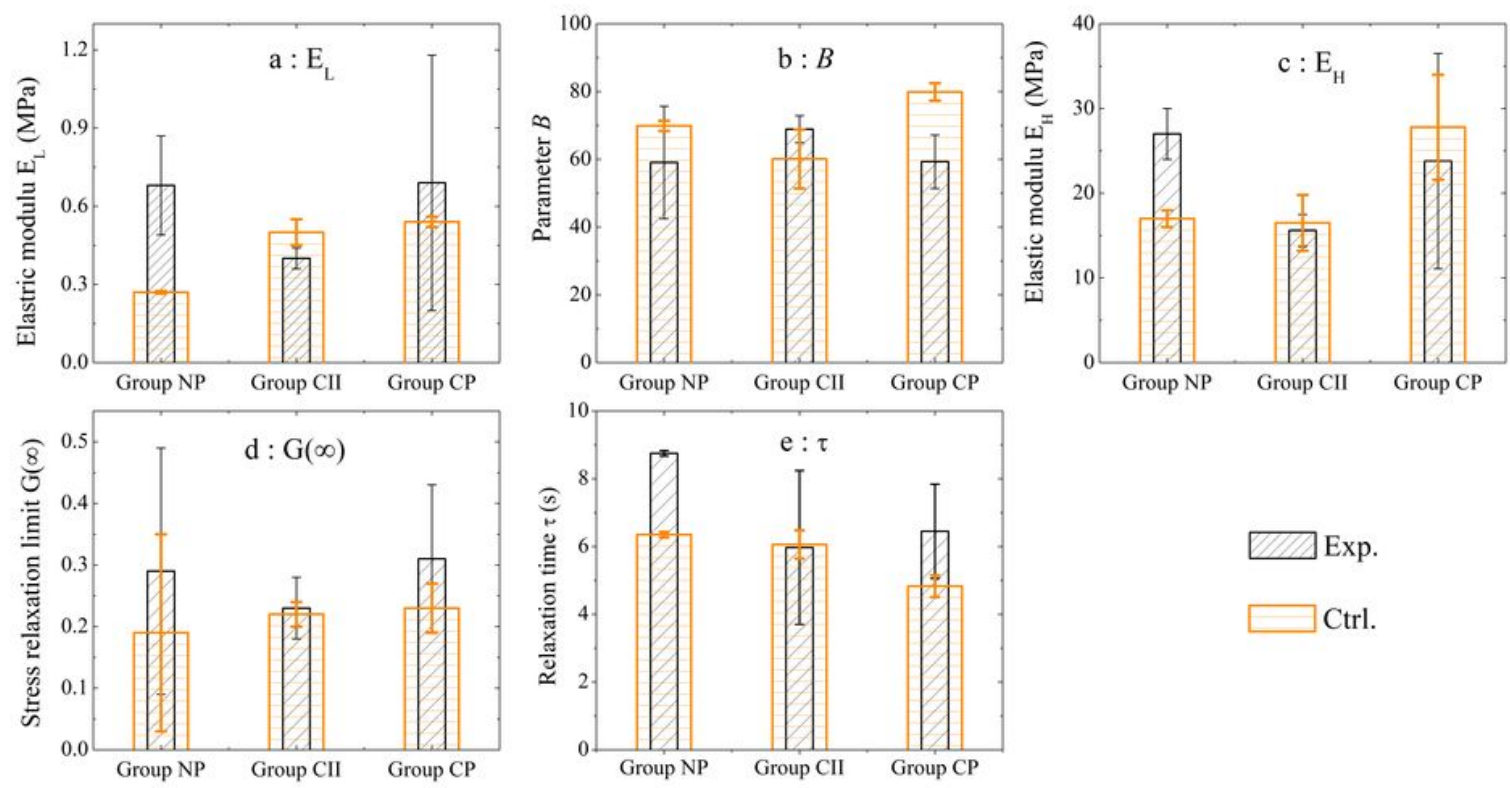

\section{Figure 6}

The biomechanical parameters of cornea in three groups. (a) and (c) are the results of the elastic modulus in the low and high stress region of strain-stress curves, (b) is the results of parameter $B$ in the nonlinear region of strain-stress curves, (d) and (e) are the results of stress relaxation limit and relaxation time of corneas. In group NP, elastic modulus at low and high stress region (EL and EH, a and c) increased obviously, while conversely in parameter B (b). And the stress relaxation time (e) increased, which indicated that the relaxation stress of experimental corneas became slow down. In both Group Cll and $\mathrm{CP}$, no significant difference was observed in all the mechanical parameters between experimental and control corneas, and the differences in Group CP were slightly larger than those in Group Cll. 


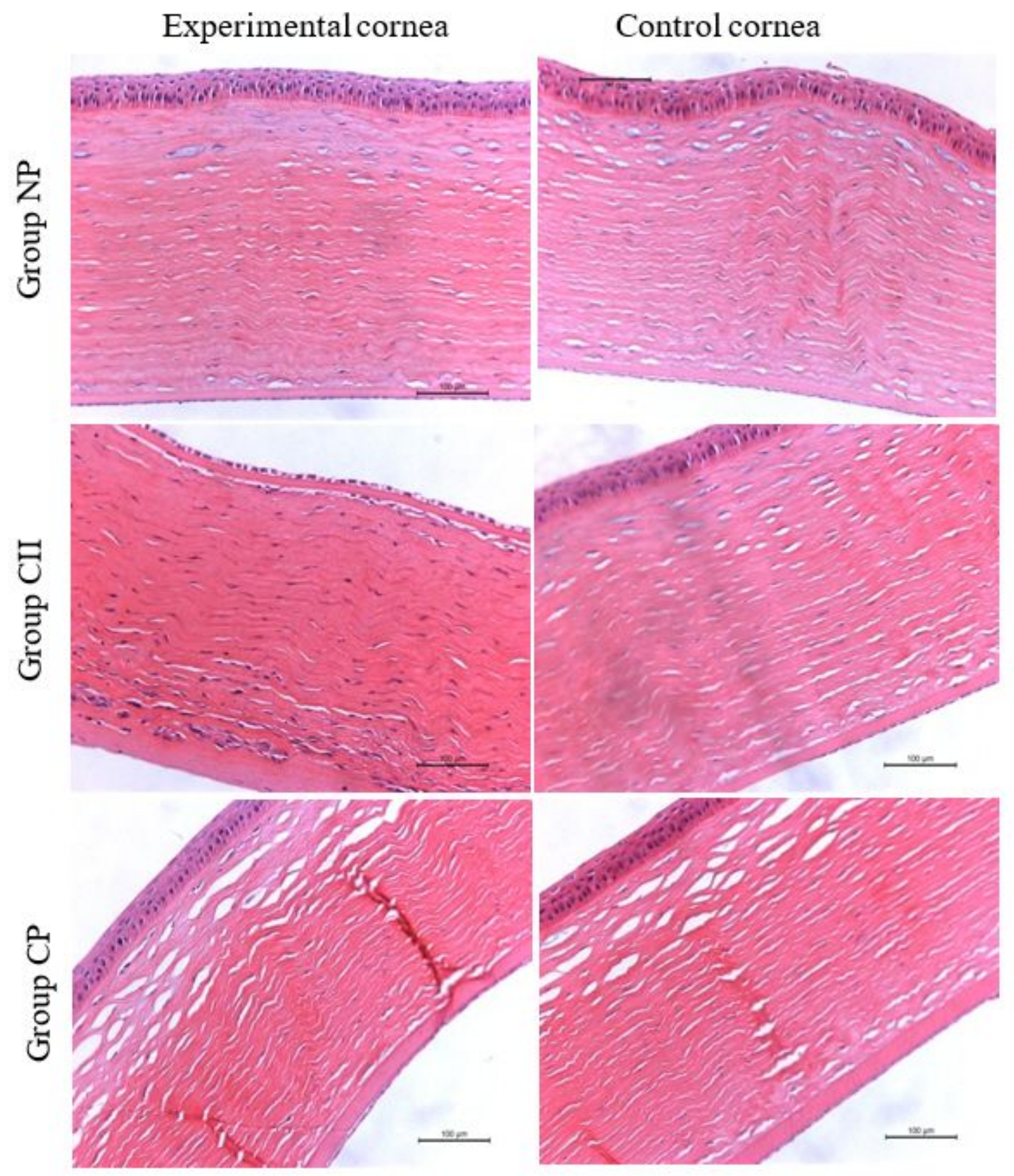

Figure 7

Hematoxylin-eosin stained corneal sections. The structure of each layer was intact and no obvious abnormality in the cell morphology was observed in all group. Some epithelial cells were lost due to sectioning. No significant difference between the experimental and control corneas in Group NP (the first row), while the experimental corneas tissue sections showed loose and disordered collagenous fibers, widened interlamellar clefts compared with control corneas in Group Cll and Group CP (the second and third row).

\section{Supplementary Files}


This is a list of supplementary files associated with this preprint. Click to download.

- NC3RsBMC.2.pdf 